\title{
A METRIC THAT BETTER APPROXIMATES THE HYPERBOLIC METRIC
}

\author{
WILLIAM MA
}

\begin{abstract}
We introduce a metric, called the star metric, that gives us better upper bound of the hyperbolic metric than the Möbius invariant metric. We present various examples, and comparisons among the hyperbolic metric, the Möbius invariant metric and the star metric.
\end{abstract}

\section{INTRODUCTION}

Let $\Omega$ be a hyperbolic region in the complex plane $\mathbb{C}$, that is $\mathbb{C} \backslash \Omega$ contains at least two points. The hyperbolic metric on $\Omega$ is denoted by $\lambda_{\Omega}(w)|d w|$ and is normalized to have curvature

$$
K\left(w, \lambda_{\Omega}\right)=-\frac{\Delta \log \lambda_{\Omega}(w)}{\lambda_{\Omega}^{2}(w)}=-1,
$$

where $w=u+i v$ and

$$
\Delta=\frac{\partial^{2}}{\partial u^{2}}+\frac{\partial^{2}}{\partial v^{2}}=4 \frac{\partial^{2}}{\partial w \partial \bar{w}}
$$

denotes the usual Laplacian. We denote the hyperbolic distance between $a, b \in \Omega$ by $d_{\Omega}(a, b)$.

In particular, the hyperbolic metric on the unit disk $\mathbb{D}=\{z:|z|<1\}$ is

$$
\lambda_{\mathbb{D}}(z)|d z|=\frac{2|d z|}{1-|z|^{2}} .
$$

The hyperbolic geodesics in $\mathbb{D}$ are circular arcs orthogonal with the unit circle and

$$
d_{\mathbb{D}}(a, b)=2 \tanh ^{-1}\left|\frac{a-b}{1-\bar{a} b}\right| .
$$

The hyperbolic metric is invariant under conformal mappings, that is, $\lambda_{\Delta}(f(z))\left|f^{\prime}(z)\right|=\lambda_{\Omega}(z)$ if $f: \Omega \rightarrow \Delta$ is conformal. In particular, if $f: \mathbb{D} \rightarrow \Omega$ is any universal covering projection, then the density $\lambda_{\Omega}$ of the hyperbolic metric is determined from $\lambda_{\Omega}(f(z))\left|f^{\prime}(z)\right|=2 /\left(1-|z|^{2}\right)$. Similarly, if $g(w): \Omega \rightarrow \mathbb{D}$ is a covering projection, then $\lambda_{\Omega}(w)=2\left|g^{\prime}(w)\right| /\left(1-|g(w)|^{2}\right)$. The domain monotonicity property, $\lambda_{\Omega}(z) \geq \lambda_{\Delta}(z)$ when $\Omega \subseteq \Delta$ with strict inequality unless $\Omega=\Delta$, is a direct consequence of Schwarz's Lemma; see [1].

Suppose $\Omega$ is a proper subregion of the complex plane $\mathbb{C}$. For any $z \in \Omega$, let $\delta_{\Omega}(z)=\operatorname{dist}(z, \partial \Omega)$. Twice of the quasi-hyperbolic metric $|d z| / \delta_{\Omega}(z)$ provides an upper bound of the hyperbolic metric $\lambda_{\Omega}(z)|d z|$ and is invariant under affine transformations.

Received by the editors March 29, 2021.

2020 Mathematics Subject Classification. Primary 30F45; Secondary 30C55, 30C20.

Key words and phrases. Hyperbolic metric, Möbius invariant metric, star metric. 
Kulkarni and Pinkall [10] introduced a canonical metric for Möbius structures and presented various properties and applications of the metric. They considered Möbius structures on n-dimensional manifolds. In [7, [8] and [9], Herron, Ma and Minda studied the corresponding metric, which we call a Möbius invariant metric denoted by $\mu_{\Omega}(z)|d z|$, for regions on the Riemann sphere $\mathbb{P}$ with at least two boundary points from classical function theory point of view. The density $\mu_{\Omega}(z)$ of the Möbius invariant metric $\mu_{\Omega}(z)|d z|$ is defined as follows.

$$
\mu_{\Omega}(z)=\inf \left\{\lambda_{D}(z): z \in D \subseteq \Omega, D \text { is a disk on } \mathbb{P}\right\} .
$$

The infinmum in this definition can be replaced by minimum [7. Herron, Ibragimov and Minda 6] studied geodesics and curvature of $\mu_{\Omega}(z)|d z|$. Also, see [4].

On one hand, the metric $\mu_{\Omega}(z)|d z|$ provides a better upper bound of the hyperbolic metric than the quasihyperbolic metric. More precisely, for any hyperbolic region $\Omega$ in the complex plane $\mathbb{C}$,

$$
\lambda_{\Omega}(z) \leq \mu_{\Omega}(z) \leq \frac{2}{\delta_{\Omega}(z)} .
$$

On the other hand, determining $\mu_{\Omega}(z)$ requires that we find the extremal disk $D$ as the definition involves a minimum.

In this paper, we introduce another metric that gives us better upper bound of the hyperbolic metric and does not involve any infinmum nor any minimum.

Let $\Omega$ be a proper subregion of $\mathbb{C}$. For each $z \in \Omega$, set

$$
\Omega^{*}(z)=\{w \in \Omega:[z, w] \subset \Omega\},
$$

where $[z, w]$ denotes the line segment between $z$ and $w$. That is, $\Omega^{*}(z)$ is the largest subregion of $\Omega$ that is starlike with respect to $z$. We define a metric $\lambda_{\Omega}^{*}(z)|d z|$ on $\Omega$ that we call star metric as follows:

$$
\lambda_{\Omega}^{*}(z)|d z|=\lambda_{\Omega^{*}(z)}(z)|d z| .
$$

It is clear from the domain monotonicity property of the hyperbolic metric that for any hyperbolic region $\Omega$ in $\mathbb{C}$,

$$
\lambda_{\Omega}(z) \leq \lambda_{\Omega}^{*}(z) \leq \mu_{\Omega}(z) \leq \frac{2}{\delta_{\Omega}(z)} .
$$

The following simple facts about the star metric $\lambda_{\Omega}^{*}(z)|d z|$ follow immediately from the definition.

(I) $\lambda_{\Omega}^{*}(z)=\lambda_{\Omega}(z)$ at any point $z \in \Omega$ if and ony if $\Omega$ is starlike with respect to $z$. In particular, for any convex region $\Omega, \lambda_{\Omega}^{*}(z)|d z|=\lambda_{\Omega}(z)|d z|$;

(II) $\lambda_{\Omega}^{*}(z)|d z|$ is monotonic with respect to the domain $\Omega$. Precisely, if $\Omega \subseteq \Delta$, then $\lambda_{\Omega}^{*}(z) \geq \lambda_{\Delta}^{*}(z)$;

(III) $\lambda_{\Omega}^{*}(z)|d z|$ is invariant under affine transformations.

In this paper, we obtain connections among the star metric $\lambda_{\Omega}^{*}(z)|d z|$ and the hyperbolic and quasi-hyperbolic metrics on hyperbolic regions in the complex plane $\mathbb{C}$. We show that $\lambda_{\Omega}^{*}(z)|d z|$ provides a better approximation for the hyperbolic metric than the quasi-hyperbolic metric and the Möbius invariant metric.

We present various comparisons among the hyperbolic metric, the quasi-hyperbolic metric, the Möbius invariant metric and $\lambda_{\Omega}^{*}(z)|d z|$. In particular, we provide estimates for $\inf \left\{\lambda_{\Omega}(z) / \lambda_{\Omega}^{*}(z): z \in \Omega\right\}$ and $\sup \left\{\lambda_{\Omega}(z) / \lambda_{\Omega}^{*}(z): z \in \Omega\right\}$. Clearly, $0 \leq$ 
$\inf \left\{\lambda_{\Omega}(z) / \lambda_{\Omega}^{*}(z): z \in \Omega\right\} \leq \sup \left\{\lambda_{\Omega}(z) / \lambda_{\Omega}^{*}(z): z \in \Omega\right\} \leq 1$. Moreover, if $\Omega$ is convex, then $\inf \left\{\lambda_{\Omega}(z) / \lambda_{\Omega}^{*}(z): z \in \Omega\right\}=1$. Otherwise, $\inf \left\{\lambda_{\Omega}(z) / \lambda_{\Omega}^{*}(z): z \in \Omega\right\}<$ 1.

\section{Preliminaries}

First, we present some examples.

Example 2.1. For $a \in \mathbb{C}, \Omega=\mathbb{C} \backslash\{a\}$, we simply write $\lambda_{\Omega}^{*}(z)$ as $\lambda_{a}^{*}(z)$ :

$$
\lambda_{a}^{*}(z)|d z|=\frac{|d z|}{2|z-a|} .
$$

As a comparison, note that for $\Omega=\mathbb{C} \backslash\{a\}[7]$,

$$
\mu_{\Omega}(z)|d z|=\frac{|d z|}{|z-a|} .
$$

Without loosing of generality, we may assume that $a=0$. Then for any $w \neq 0$,

$$
\begin{gathered}
\Omega^{*}(w)=\mathbb{C} \backslash\{-t w: t \geq 0\}, \\
g(z)=w\left(\frac{1+z}{1-z}\right)^{2}: \mathbb{D} \rightarrow \Omega^{*}(w)
\end{gathered}
$$

is a covering projection, and $\lambda_{0}^{*}(w)=\frac{2}{\left|g^{\prime}(0)\right|}=\frac{1}{2|w|}$.

Example 2.2. For $\Omega=\mathbb{D} \backslash\{0\}$,

$$
\lambda_{\Omega}^{*}(z)=\frac{1+|z|}{2|z|(1-|z|)} .
$$

In particular, $\lim _{|z| \rightarrow 0} \frac{\lambda_{\Omega}(z)}{\lambda_{\Omega}^{*}(z)}=0$ and $\lim _{|z| \rightarrow 1} \frac{\lambda_{\Omega}(z)}{\lambda_{\Omega}^{*}(z)}=1$. Also, note that $\mu_{\Omega}(z)=$ $\frac{1}{|z|(1-|z|)}[7]$.

We only need to consider those $x \in(0,1) . \Omega^{*}(x)=\mathbb{D} \backslash(-1,0)$. By using the composition of the following maps $\eta=\sqrt{z}, \zeta=\frac{\eta-i}{\eta+i}, \xi=\zeta^{2}$, and $w=\frac{1+i}{1-i} \frac{\xi-i}{\xi+i}$, we get a covering projection

$$
g(z)=\frac{1-z-2 \sqrt{z}}{1-z+2 \sqrt{z}}: \Omega^{*}(x) \rightarrow \mathbb{D}
$$

where $\sqrt{z}$ takes postive value when $z>0$.

Direct calculations give us

$$
g^{\prime}(z)=\frac{-2(1+z)}{\sqrt{z}(1-z+2 \sqrt{z})^{2}}
$$

and

$$
\lambda_{\Omega}^{*}(x)=\lambda_{\Omega^{*}(x)}(x)=\frac{2\left|g^{\prime}(x)\right|}{1-|g(x)|^{2}}=\frac{1+x}{2 x(1-x)},
$$

which implies the expression for $\lambda_{\Omega}^{*}(z)$.

Combining the expression of $\lambda_{\Omega}^{*}(z)$ with $\lambda_{\Omega}(z)=\frac{1}{|z| \log (1 /|z|)}$, we can easily get the other results.

Similar as the example above, $\inf \left\{\lambda_{\Omega}(z) / \lambda_{\Omega}^{*}(z): z \in \Omega\right\}=0$ for any hyperbolic region $\Omega$ with an isolated boundary point. This can be seen by estimating the hyperbolic metric near an isolated point. 
Example 2.3. Let $\Omega_{\alpha}=\{w:|\arg w|<\pi \alpha / 2\}, 1<\alpha \leq 2$. Then

$$
\lambda_{\Omega_{\alpha}}^{*}\left(r e^{i \theta}\right)= \begin{cases}\frac{1}{\alpha r \cos (\theta / \alpha)} & \text { if }|\theta| \leq \pi(1-\alpha / 2), \\ \frac{\pi}{r(\pi-|\theta|+\pi \alpha / 2) \cos \left(\frac{\pi}{2} \frac{\pi+|\theta|-\pi \alpha / 2}{\pi-|\theta|+\pi \alpha / 2}\right)} & \text { if } \pi(1-\alpha / 2)<|\theta|<\pi \alpha / 2 .\end{cases}
$$

Moreover,

$$
\frac{\lambda_{\Omega_{\alpha}}\left(r e^{i \theta}\right)}{\lambda_{\Omega_{\alpha}}^{*}\left(r e^{i \theta}\right)}= \begin{cases}1 & \text { if }|\theta| \leq \pi(1-\alpha / 2), \\ \frac{(\pi-|\theta|+\pi \alpha / 2) \cos \left(\frac{\pi}{2} \frac{\pi+|\theta|-\pi \alpha / 2}{\pi-|\theta|+\pi \alpha / 2}\right)}{\pi \alpha \cos (\theta / \alpha)} & \text { if } \pi(1-\alpha / 2)<|\theta|<\pi \alpha / 2 .\end{cases}
$$

In particular, for each $1<\alpha \leq 2$,

$$
\lim _{|\theta| \rightarrow \pi \alpha / 2} \frac{\lambda_{\Omega_{\alpha}}\left(r e^{i \theta}\right)}{\lambda_{\Omega_{\alpha}}^{*}\left(r e^{i \theta}\right)}=1 .
$$

Since $f(z)=\left(\frac{1+z}{1-z}\right)^{\alpha}: \mathbb{D} \rightarrow \Omega_{\alpha}$ is a covering, it is straightforward to calculate that

$$
\lambda_{\Omega_{\alpha}}\left(r e^{i \theta}\right)=\frac{1}{\alpha r \cos (\theta / \alpha)}
$$

by using $\lambda_{\Omega_{\alpha}}(f(z))\left|f^{\prime}(z)\right|=\lambda_{\mathbb{D}}(z)=2 /\left(1-|z|^{2}\right)$.

Next, we determine $\lambda_{\Omega_{\alpha}}^{*}\left(r e^{i \theta}\right)$ in three cases.

For $a=r e^{i \theta}$ with $|\theta| \leq \pi(1-\alpha / 2), \Omega_{\alpha}^{*}(a)=\Omega_{\alpha}$, so $\lambda_{\Omega_{\alpha}}^{*}(a)=\lambda_{\Omega_{\alpha}}(a)$.

The case of $-\pi \alpha / 2<\theta<-\pi(1-\alpha / 2)$ follows from the next case by using symmetry.

Now, we consider the case when $\pi(1-\alpha / 2)<\theta<\pi \alpha / 2$. In this case, $\Omega_{\alpha}^{*}(a)$ is an infinite sector with angle of opening $2 \pi-\theta-\pi(1-\alpha / 2)=\pi-\theta+\pi \alpha / 2$ and center half line $\left\{t e^{i \phi}: t>0\right\}$, where $\phi=-(\pi-\theta)+\frac{1}{2}(\pi-\theta+\pi \alpha / 2)=\pi \alpha / 4-(\pi-\theta) / 2$. Hence,

$$
g(z)=\left[z e^{i\left(\frac{\pi-\theta}{2}-\frac{\pi \alpha}{4}\right)}\right]^{\frac{\pi}{\pi-\theta+\pi \alpha / 2}}=\exp \left\{i \frac{\pi}{2} \frac{\pi-\theta-\pi \alpha / 2}{\pi-\theta+\pi \alpha / 2}\right\} z^{\frac{\pi}{\pi-\theta+\pi \alpha / 2}}
$$

is a conformal map from $\Omega_{\alpha}^{*}(a)$ to the right half plane $\mathbb{H}$. As $\lambda_{\mathbb{H}}(w)=1 / \operatorname{Re} w$,

$$
\lambda_{\Omega_{\alpha}^{*}(a)}(z)=\frac{\left|g^{\prime}(z)\right|}{\operatorname{Re} g(z)}=\frac{\pi|z|^{\frac{\pi}{\pi-\theta-\pi \alpha / 2}}-1}{(\pi-\theta+\pi \alpha / 2) \operatorname{Re}\left\{\exp \left\{i \frac{\pi}{2} \frac{\pi-\theta-\pi \alpha / 2}{\pi-\theta+\pi \alpha / 2}\right\} z^{\frac{\pi}{\pi-\theta+\pi \alpha / 2}}\right\}} .
$$

In particular, at $z=r e^{i \theta}$, we get

$$
\begin{aligned}
\lambda_{\Omega_{\alpha}}^{*}\left(r e^{i \theta}\right) & =\frac{\pi}{r\left(\pi-\theta+\frac{\pi \alpha}{2}\right) \cos \left(\frac{\pi}{2} \frac{\pi-\theta-\pi \alpha / 2}{\pi-\theta+\pi \alpha / 2}+\frac{\pi \theta}{\pi-\theta+\pi \alpha / 2}\right)} \\
& =\frac{\pi}{r\left(\pi-\theta+\frac{\pi \alpha}{2}\right) \cos \left(\frac{\pi}{2} \frac{\pi+\theta-\pi \alpha / 2}{\pi-\theta+\pi \alpha / 2}\right)} .
\end{aligned}
$$

Combining the expressions for $\lambda_{\Omega_{\alpha}}\left(r e^{i \theta}\right)$ and $\lambda_{\Omega_{\alpha}}^{*}\left(r e^{i \theta}\right)$, it is straightforward to derive the desired results.

Now, we show that the star metric and the quasi-hyperbolic metric are equivalent.

Theorem 2.4. For any proper subregion $\Omega$ of $\mathbb{C}$,

$$
\frac{1}{2 \delta_{\Omega}(z)} \leq \lambda_{\Omega}^{*}(z) \leq \frac{2}{\delta_{\Omega}(z)} .
$$


Both constants are best possible. Moreover, $\Omega$ is uniformly perfect if and only if

$$
\inf \left\{\lambda_{\Omega}(z) / \lambda_{\Omega}^{*}(z): z \in \Omega\right\}>0 .
$$

Proof. We only need to prove the first inequality for any fixed $z \in \Omega$. Set $\delta=\delta_{\Omega}(z)$. From Theorem 4 in [12], $S_{\Omega}(z) \geq \frac{1}{4 \delta_{\Omega}(z)}$, where $S_{\Omega}(z)|d z|$ is the Hahn metric. The desired inequality follows from the obvious fact that $\lambda_{\Omega}(z) \leq 2 S_{\Omega}(z) \leq \lambda_{\Omega}^{*}(z)$, where the extra factor 2 is due to a different normalization of the Hahn metric in 12 .

Example 2.1 tells us that the constant $1 / 2$ in the lower bound cannot be improved, while at 0 in $\mathbb{D}$ the equality in the upper bound is achieved. The last statement follows from the fact that $\Omega$ is uniformly perfect if and only if

$$
\inf \left\{\lambda_{\Omega}(z) \delta_{\Omega}(z): z \in \Omega\right\}>0 .
$$

Similarly we can compare the Möbius invariant metric $\mu_{\Omega}(z)|d z|$ and the star metric $\lambda_{\Omega}^{*}(z)|d z|$.

Theorem 2.5. For any proper subregion $\Omega$ of $\mathbb{C}$,

$$
\frac{1}{2} \mu_{\Omega}(z) \leq \lambda_{\Omega}^{*}(z) \leq \mu_{\Omega}(z)
$$

Both constants are best possible.

Proof. The second inequality follows easily from the definitions. So we only need to prove the first inequality for any fixed $a \in \Omega$.

Let $D=D(a, \Omega)$ be the extremal disk (or half plane) for $\mu_{\Omega}(z)|d z|$ at $a$. Then $D \subseteq \Omega^{*}(a)$ and $\mu_{\Omega}(a)=\lambda_{D}(a)=\mu_{\Omega^{*}(a)}(a)$. As $\lambda_{\Omega}^{*}(a)=\lambda_{\Omega^{*}(a)}(a)$, it is enough that we show $\frac{1}{2} \mu_{\Omega^{*}(a)}(a) \leq \lambda_{\Omega^{*}(a)}(a)$, which follows from the fact that $\frac{1}{2} \mu_{\Delta}(z) \leq \lambda_{\Delta}(z)$ for any simply connected region $\Delta$; see 7 .

For $\Omega=\mathbb{C} \backslash(-\infty, 0]$ and $x>0, \mu_{\Omega}(x)=\lambda_{\mathbb{H}}(x)=1 / x$, where $\mathbb{H}$ is the right half plane, while $\Omega^{*}(x)=\Omega$ and $\lambda_{\Omega}^{*}(x)=\lambda_{\Omega}(x)=1 /(2 x)$. Thus, $\frac{1}{2} \mu_{\Omega}(x)=\lambda_{\Omega}^{*}(x)$.

Next, we show that $\lambda_{\Omega}^{*}$ depends continuously on $\Omega$. We write $\Omega_{n} \rightarrow \Omega$ to mean that the sequence $\left\{\Omega_{n}\right\}$ converges to $\Omega$ in the sense of kernel convergence with respect to some fixed point. Recall that $\lim _{n \rightarrow \infty} \lambda_{\Omega_{n}}(z)=\lambda_{\Omega}(z)$ when $\Omega_{n} \rightarrow \Omega[5]$.

Theorem 2.6. Suppose $\left\{\Omega_{n}\right\}_{n=1}^{\infty}$ is a sequence of hyperbolic regions in $\mathbb{C}$ and $\Omega_{n} \rightarrow \Omega$. Then for each $z \in \Omega, \lim _{n \rightarrow \infty} \lambda_{\Omega_{n}}^{*}(z)=\lambda_{\Omega}^{*}(z)$.

Proof. Fix $a \in \Omega$. We first prove that

$$
\lim \sup _{n \rightarrow \infty} \lambda_{\Omega_{n}}^{*}(a) \leq \lambda_{\Omega}^{*}(a) .
$$

For any $t<1$, let $\Delta_{t}=\left\{a+t(z-a): z \in \Omega^{*}(a)\right\}$. Then $\overline{\Delta_{t}}$ is a compact subset of $\Omega^{*}(a) \subseteq \Omega$. So there exists $N$ such that $\overline{\Delta_{t}} \subset \Omega_{n}$ for all $n \geq N$. Note that $\Delta_{t}$ is starlike with respect to $a$. Hence, for $n \geq N, \Delta_{t} \subseteq \Omega_{n}^{*}(a)$ and $\lambda_{\Omega_{n}}^{*}(a) \leq \lambda_{\Delta_{t}}(a)$, so

$$
\lim \sup _{n \rightarrow \infty} \lambda_{\Omega_{n}}^{*}(a) \leq \lambda_{\Delta_{t}}(a) .
$$

Choose $\left\{t_{n}\right\}$ be an increasing sequence with limit 1. As $\lim _{n \rightarrow \infty} \lambda_{\Delta_{t_{n}}}(a)=\lambda_{\Omega^{*}(a)}(a)$, by letting $t_{n} \rightarrow 1$, we obtain

$$
\lim \sup _{n \rightarrow \infty} \lambda_{\Omega_{n}}^{*}(a) \leq \lambda_{\Omega^{*}(a)}(a)=\lambda_{\Omega}^{*}(a) .
$$

Next, we prove that

$$
m=\liminf _{n \rightarrow \infty} \lambda_{\Omega_{n}}^{*}(a) \geq \lambda_{\Omega}^{*}(a) .
$$


By passing to an appropriate subsequence of $\Omega_{n}$ if necessary, we may assume $\lim _{n \rightarrow \infty} \lambda_{\Omega_{n}}^{*}(a)=m$. Consider conformal mappings $g_{n}: \mathbb{D} \rightarrow \Omega_{n}^{*}(a)$ such that $g_{n}(0)=a$ and $g_{n}^{\prime}(0)>0$. Then

$$
\lambda_{\Omega_{n}}^{*}(a)=\lambda_{\Omega_{n}^{*}(a)}(a)=\frac{2}{g_{n}^{\prime}(0)},
$$

or equivalently, $g_{n}^{\prime}(0)=2 / \lambda_{\Omega_{n}}^{*}(a)$. From $\Omega_{n} \rightarrow \Omega, \lambda_{\Omega_{n}}(a) \rightarrow \lambda_{\Omega}(a)$ [5]. Because $\lambda_{\Omega_{n}}(a) \leq \lambda_{\Omega_{n}}^{*}(a)$, we obtain

$$
0<\lambda_{\Omega}(a)=\lim _{n \rightarrow \infty} \lambda_{\Omega_{n}}(a) \leq \lim _{n \rightarrow \infty} \lambda_{\Omega_{n}}^{*}(a)=m .
$$

Also, from what we have proved in the other direction, we see that

$$
m=\lim _{n \rightarrow \infty} \lambda_{\Omega_{n}}^{*}(a) \leq \lim \sup _{n \rightarrow \infty} \lambda_{\Omega_{n}}^{*}(a) \leq \lambda_{\Omega}^{*}(a) .
$$

So,

$$
0<\lambda_{\Omega}(a) \leq m \leq \lambda_{\Omega}^{*}(a)<\infty .
$$

As $\lim _{n \rightarrow \infty} \lambda_{\Omega_{n}}^{*}(a)=m \neq 0, \infty$, there exists $M$ so that $1 / M \leq g_{n}^{\prime}(0) \leq M$ for all $n$. This implies that the sequence $\left\{g_{n}\right\}_{n=1}^{\infty}$ is a normal family, so we may assume $g_{n} \rightarrow g$ locally uniformally on $\mathbb{D}$. The limit function $g$ is not a constant since $1 / M \leq g^{\prime}(0) \leq M$. Then $g$ is a conformal mapping with $g(0)=a, g^{\prime}(0)>0$ and $g(\mathbb{D})$ is starlike with respect to $a=g(0)$. Because $2 / \lambda_{\Omega_{n}}^{*}(a)=g_{n}^{\prime}(0) \rightarrow g^{\prime}(0)$, we must have $g^{\prime}(0)=2 / m$.

Since $g_{n}(\mathbb{D})=\Omega_{n}^{*}(a) \subseteq \Omega_{n}, \Omega_{n} \rightarrow \Omega$ and $g_{n} \rightarrow g$, it follows that $g(\mathbb{D}) \subseteq \Omega$, which then implies $g(\mathbb{D}) \subseteq \Omega^{*}(a)$. Hence,

$$
\lambda_{\Omega}^{*}(a)=\lambda_{\Omega^{*}(a)}(a) \leq \lambda_{g(\mathbb{D})}(a)=\frac{2}{g^{\prime}(0)}=m .
$$

\section{Estimates ON $\lambda_{\Omega}(z) / \lambda_{\Omega}^{*}(z)$}

For a hyperbolic region $\Omega$ in $\mathbb{C}$, following [3], we set

$$
\eta(\Omega)=2 \sup \left\{\frac{\left|\frac{\partial}{\partial w} \log \lambda_{\Omega}(w)\right|}{\lambda_{\Omega}(w)}: w \in \Omega\right\} .
$$

If $f: \mathbb{D} \rightarrow \Omega$ is any covering projection, then

$$
\eta(\Omega)=\alpha(f):=\frac{1}{2} \sup \left\{\left|\left(1-|z|^{2}\right) \frac{f^{\prime \prime}(z)}{f^{\prime}(z)}-2 \bar{z}\right|: z \in \mathbb{D}\right\} .
$$

Note that $\alpha(f)$ is called the linearly invariant order of $f$, which was introduced by Pommerenke [13; also see [11].

We denote by $D_{\mathbb{D}}(a, \rho)$ the hyperbolic disk in $\mathbb{D}$ with hyperbolic center $a$ and hyperbolic redius $\rho$. The hyperbolic radius of uniform local covexity of a holomorphic function $f$ in $\mathbb{D}$ is defined by

$\rho_{c}(f)=\sup \left\{\rho: f\right.$ is univalent in $D_{\mathbb{D}}(a, \rho)$ and $f\left(D_{\mathbb{D}}(a, \rho)\right)$ is convex for all a $\left.\in \mathbb{D}\right\}$.

Similarly, the hyperbolic radius of uniform local starlikeness of a holomorphic function $f$ in $\mathbb{D}$ is defined by

$$
\rho^{*}(f)=\sup \left\{\begin{array}{l}
\rho: f \text { is univalent in } D_{\mathbb{D}}(a, \rho) \text { and } \\
f\left(D_{\mathbb{D}}(a, \rho)\right) \text { is starlike with respect to } f(a) \text { for all } a \in \mathbb{D}
\end{array}\right\} .
$$


Lemma 3.1. For any holomorphic function $f$ in $\mathbb{D}, \rho^{*}(f) \geq 2 \rho_{c}(f)$.

Proof. Set $\rho=2 \rho_{c}(f)$. First, we prove that $f$ is univalent in any hyperbolic disk $D_{\mathbb{D}}(a, \rho)$. If not, there exist $b, c \in D_{\mathbb{D}}(a, \rho)$ such that $f(b)=f(c)$. Let $\rho_{1}=$ $(1 / 4) d_{\mathbb{D}}(b, c)$ and $a_{1}, d, a_{2}$ be points on the hyperbolic geodesic connecting $b$ and $c$ such that $d_{\mathbb{D}}\left(b, a_{1}\right)=d_{\mathbb{D}}\left(a_{1}, d\right)=d_{\mathbb{D}}\left(d, a_{2}\right)=d_{\mathbb{D}}\left(a_{2}, c\right)$. As $d_{\mathbb{D}}(b, c)<2 \rho, \rho_{1}<$ $\rho_{c}(f), f$ is univalent in $\overline{D_{\mathbb{D}}\left(a_{1}, \rho_{1}\right)}$ and $\overline{D_{\mathbb{D}}\left(a_{2}, \rho_{1}\right)}$, respectively, and $f\left(\overline{D_{\mathbb{D}}\left(a_{1}, \rho_{1}\right)}\right)$, $f\left(\overline{D_{\mathbb{D}}\left(a_{2}, \rho_{1}\right)}\right)$ are convex. Since $f(d), f(b)=f(c) \in f\left(\overline{D_{\mathbb{D}}\left(a_{1}, \rho_{1}\right)}\right) \cap f\left(\overline{D_{\mathbb{D}}\left(a_{2}, \rho_{1}\right)}\right)$, the Euclidean line segment $[f(d), f(b)] \subseteq f\left(\overline{D_{\mathbb{D}}\left(a_{1}, \rho_{1}\right)}\right) \cap f\left(\overline{D_{\mathbb{D}}\left(a_{2}, \rho_{1}\right)}\right)$. Then for a point $A$ close enough to $f(d)$ on $[f(d), f(b)]$, there exists $z_{1} \in \overline{D_{\mathbb{D}}\left(a_{1}, \rho_{1}\right)} \backslash\{d\}$, $z_{2} \in \overline{D_{\mathbb{D}}\left(a_{2}, \rho_{1}\right)} \backslash\{d\}$ such that $0<d_{\mathbb{D}}\left(z_{1}, z_{2}\right)<\rho_{c}(f)$, and $f\left(z_{1}\right)=f\left(z_{2}\right)=A$. This contradicts to the fact that $f$ is univalent in any hyperbolic disk of radius $\rho_{c}(f)$ as $z_{1} \neq z_{2}$, which follows from that $\overline{D_{\mathbb{D}}\left(a_{1}, \rho_{1}\right)} \backslash\{d\}$ and $\overline{D_{\mathbb{D}}\left(a_{2}, \rho_{1}\right)} \backslash\{d\}$ do not intersect each other.

Next, we show that for any fixed hyperbolic disk $D_{\mathbb{D}}(a, \rho), f\left(D_{\mathbb{D}}(a, \rho)\right)$ is starlike with respect to $f(a)$. For each $b \in D_{\mathbb{D}}(a, \rho)$, let $c$ be the point on the hyperbolic geodesic connecting $a$ and $b$ such that $d_{\mathbb{D}}(a, c)=d_{\mathbb{D}}(b, c)=d_{\mathbb{D}}(a, b) / 2$. Then $a, b \in D_{\mathbb{D}}(c, \rho / 2)$. Since $f\left(D_{\mathbb{D}}(c, \rho / 2)\right)$ is convex, the line segment $[f(a), f(b)]$ lies in $f\left(D_{\mathbb{D}}(c, \rho / 2)\right)$. As $D_{\mathbb{D}}(c, \rho / 2) \subset D_{\mathbb{D}}(a, \rho),[f(a), f(b)] \subset f\left(D_{\mathbb{D}}(a, \rho)\right)$. Because $b \in D_{\mathbb{D}}(a, \rho)$ is arbitrary, we see that $f\left(D_{\mathbb{D}}(a, \rho)\right)$ is starlike with respect to $f(a)$.

Therefore, $\rho^{*}(f) \geq \rho=2 \rho_{c}(f)$.

Theorem 3.2. Suppose $\Omega$ is a hyperbolic region in $\mathbb{C}$. Then

$$
\frac{\lambda_{\Omega}(z)}{\lambda_{\Omega}^{*}(z)} \geq \frac{1}{\eta(\Omega)} .
$$

Proof. Set $\eta=\eta(\Omega)$. For any $a \in \Omega$, let $f: \mathbb{D} \rightarrow \Omega$ be a covering projection with $f(0)=a$. Then $\alpha(f)=\eta$. We also know that $[11] \alpha(f)=\operatorname{coth}\left(\rho_{c}(f)\right)$. By using Lemma 3.1, we have

$$
R:=\tanh \frac{\rho^{*}(f)}{2} \geq \tanh \rho_{c}(f)=\frac{1}{\alpha(f)}=\frac{1}{\eta} .
$$

Thus, $f(|z|<R)$ is starlike with respect to $f(0)=a$, which implies that $\Delta=f(|z|<$ $R) \subset \Omega^{*}(a)$ and $\lambda_{\Omega}^{*}(a) \leq \lambda_{\Delta}(a)$. Since $\lambda_{\Omega}(a)=2 /\left|f^{\prime}(0)\right|$ and $\lambda_{\Delta}(a)=2 /\left(R\left|f^{\prime}(0)\right|\right)$, we have

$$
\frac{\lambda_{\Omega}(a)}{\lambda_{\Omega}^{*}(a)} \geq \frac{\lambda_{\Omega}(a)}{\lambda_{\Delta}(a)}=R \geq \frac{1}{\eta} .
$$

Suppose $\Omega$ is simply connected. Then any covering projection $f: \mathbb{D} \rightarrow \Omega$ is univalent in $\mathbb{D}$. It is well known that $\alpha(f) \leq 2$, thus $\eta(\Omega) \leq 2$. The above theorem implies that $\lambda_{\Omega}(z) / \lambda_{\Omega}^{*}(z) \geq 1 / 2$. This can be improved as follows.

Theorem 3.3. Suppose $\Omega$ is a simply connected hyperbolic region in $\mathbb{C}$. Then

$$
\frac{\lambda_{\Omega}(z)}{\lambda_{\Omega}^{*}(z)} \geq \tanh \frac{\pi}{4} \approx 0.65579 \ldots
$$

Proof. For any $a \in \Omega$, let $f: \mathbb{D} \rightarrow \Omega$ be a covering projection with $f(0)=a$. Define

$$
g(z)=\frac{f(z)-f(0)}{f^{\prime}(0)} .
$$


Then $g$ is a normalized $\left(g(0)=g^{\prime}(0)-1=0\right)$ holomorphic univalent function in $\mathbb{D}$. It is well known, see [2, that $g(|z|<\tanh (\pi / 4))$ is starlike with respect to $g(0)=0$. This implies that $\Delta:=f(|z|<\tanh (\pi / 4))$ is starlike with respect to $f(0)=a$. Thus, $\Delta \subset \Omega^{*}(a)$ and $\lambda_{\Omega}^{*}(a) \leq \lambda_{\Delta}(a)$. Note that $f(z \tanh (\pi / 4)): \mathbb{D} \rightarrow \Delta$ is a covering projection, we get

$$
\frac{\lambda_{\Omega}(z)}{\lambda_{\Omega}^{*}(z)} \geq \frac{\lambda_{\Omega}(z)}{\lambda_{\Delta}(z)}=\frac{2 /\left|f^{\prime}(0)\right|}{2 /\left(\left|f^{\prime}(0)\right| \tanh (\pi / 4)\right)}=\tanh \left(\frac{\pi}{4}\right) .
$$

Remark 3.4. In our proof above, it is clear that $f(|z|<\tanh (\pi / 4))$ is a proper subset of $\Omega^{*}(a)$. This suggests that $\tanh (\pi / 4)$ is not best possible.

\section{QUASI-INVARIANCE OF $\lambda_{\Omega}^{*}(z)|d z|$}

Suppose $\Omega$ and $\Delta$ are conformal equivalent, we know that $[8]$

$$
\frac{1}{2} \mu_{\Omega}(z) \leq \mu_{\Delta}(f(z))\left|f^{\prime}(z)\right| \leq 2 \mu_{\Omega}(z) .
$$

We now show that $\lambda_{\Omega}^{*}(z)|d z|$ is quasi-invariant under conformal mappings. Note that $\operatorname{coth}(\pi / 4)=1.52 \ldots$..

Theorem 4.1. Suppose $\Omega$ and $\Delta$ are hyperbolic regions in $\mathbb{C}$ and $f: \Omega \rightarrow \Delta$ is a conformal mapping. Then

$$
\tanh (\pi / 4) \lambda_{\Omega}^{*}(z) \leq \lambda_{\Delta}^{*}(f(z))\left|f^{\prime}(z)\right| \leq \operatorname{coth}(\pi / 4) \lambda_{\Omega}^{*}(z) .
$$

Proof. We only need to prove the upper bound. For any fixed $a \in \Omega$, let $g$ : $\mathbb{D} \rightarrow \Omega^{*}(a)$ be a conformal mapping with $g(0)=a$. Then $\lambda_{\Omega}^{*}(a)=2 /\left|g^{\prime}(0)\right|$ and $F(z)=f(g(z))$ is holomorphic and univalent in $\mathbb{D}$ with $F(0)=f(a)$. Similarly as we did in the proof of Theorem 3.3. we see that $F(|z|<\tanh (\pi / 4))$ is starlike with respect to $F(0)=f(a)$. Hence, $F(|z|<\tanh (\pi / 4)) \subset \Delta^{*}(f(a))$ and $\lambda_{\Delta}^{*}(f(a)) \leq$ $\lambda_{F(|z|<\tanh (\pi / 4))}(f(a))$. Since $F(z \tanh (\pi / 4)): \mathbb{D} \rightarrow F(|z|<\tanh (\pi / 4))$ is a covering projection, we have

$$
\lambda_{F(|z|<\tanh (\pi / 4))}(f(a))=\frac{2}{\left|F^{\prime}(0)\right| \tanh (\pi / 4)}=\frac{2}{\left|f^{\prime}(a)\right|\left|g^{\prime}(0)\right| \tanh (\pi / 4)} .
$$

Therefore,

$$
\frac{\lambda_{\Delta}^{*}(f(a))\left|f^{\prime}(a)\right|}{\lambda_{\Omega}^{*}(a)} \leq \frac{1}{\tanh (\pi / 4)}=\operatorname{coth}(\pi / 4)
$$

\section{REFERENCES}

[1] A. F. Beardon and D. Minda, The hyperbolic metric and geometric function theory, Quasiconformal mappings and their applications, Narosa, New Delhi, 2007, pp. 9-56. MR2492498

[2] Peter L. Duren, Univalent functions, Grundlehren der mathematischen Wissenschaften [Fundamental Principles of Mathematical Sciences], vol. 259, Springer-Verlag, New York, 1983. MR708494

[3] Reuven Harmelin and David Minda, Quasi-invariant domain constants, Israel J. Math. 77 (1992), no. 1-2, 115-127, DOI 10.1007/BF02808014. MR1194789

[4] Barbara B. Flinn and David A. Herron, Uniform estimates for the hyperbolic metric and Euclidean distance to the boundary, Michigan Math. J. 46 (1999), no. 1, 13-27, DOI $10.1307 / \mathrm{mmj} / 1030132355$. MR 1682884

[5] Dennis A. Hejhal, Universal covering maps for variable regions, Math. Z. 137 (1974), 7-20, DOI 10.1007/BF01213931. MR349989 
[6] David A. Herron, Zair Ibragimov, and David Minda, Geodesics and curvature of Möbius invariant metrics, Rocky Mountain J. Math. 38 (2008), no. 3, 891-921, DOI 10.1216/RMJ2008-38-3-891. MR2426525

[7] David A. Herron, William Ma, and David Minda, A Möbius invariant metric for regions on the Riemann sphere, Future trends in geometric function theory, Rep. Univ. Jyväskylä Dep. Math. Stat., vol. 92, Univ. Jyväskylä, Jyväskylä, 2003, pp. 101-118. MR2058115

[8] David A. Herron, William Ma, and David Minda, Estimates for conformal metric ratios, Comput. Methods Funct. Theory 5 (2005), no. 2, 323-345, DOI 10.1007/BF03321101. MR2205417

[9] David A. Herron, William Ma, and David Minda, Möbius invariant metrics bilipschitz equivalent to the hyperbolic metric, Conform. Geom. Dyn. 12 (2008), 67-96, DOI 10.1090/S10884173-08-00178-1. MR2410919

[10] Ravi S. Kulkarni and Ulrich Pinkall, A canonical metric for Möbius structures and its applications, Math. Z. 216 (1994), no. 1, 89-129, DOI 10.1007/BF02572311. MR.1273468

[11] Wan Cang Ma and David Minda, Euclidean linear invariance and uniform local convexity, J. Austral. Math. Soc. Ser. A 52 (1992), no. 3, 401-418. MR.1151295

[12] C. David Minda, The Hahn metric on Riemann surfaces, Kodai Math. J. 6 (1983), no. 1, 57-69, DOI 10.2996/kmj/1138036663. MR698327

[13] Christian Pommerenke, Linear-invariante Familien analytischer Funktionen I (German), Math. Ann. 155 (1964), no. 2, 108-154, DOI 10.1007/BF01344077. MR.1513275

Department of Mathematics, School of Business, Arts and Sciences, Pennsylvania

College of Technology, Williamsport, Pennsylvania 17701

Email address: wma@pct.edu 